\title{
Predictors of increasing BMI during the course of diabetes in children and adolescents with type 1 diabetes: data from the German/Austrian DPV multicentre survey
}

\author{
Elke E Fröhlich-Reiterer, ${ }^{1}$ Joachim Rosenbauer, ${ }^{2}$ Susanne Bechtold-Dalla Pozza, ${ }^{3}$ \\ Sabine E Hofer, ${ }^{4}$ Edith Schober, ${ }^{5}$ Reinhard W Holl, ${ }^{6}$ on behalf of the DPV-Wiss Study \\ Group and the German BMBF Competence Networks Diabetes mellitus and Obesity
}

- For a full list of the centres participating in the DPV initiative and contributing their data to this study please see the online supplementary file on our website (http://dx/doi. org/10.1136/archdischild2013-304237)

${ }^{1}$ Department of Paediatrics, Medical University of Graz, Graz, Austria

${ }^{2}$ Institute for Biometrics and Epidemiology, German Diabetes Center, Leibniz Institute for Diabetes Research at Heinrich Heine University Düsseldorf, Düsseldorf, Germany

${ }^{3}$ Department of Paediatrics, University Munich, Munich, Germany

${ }^{4}$ Department of Paediatrics, Medical University of Innsbruck, Innsbruck, Austria ${ }^{5}$ Department of Paediatrics, Medical University of Vienna, Vienna, Austria

${ }^{6}$ Institute of Epidemiology and Medical Biometry, University of Ulm, Ulm, Germany

\section{Correspondence to Dr Elke E Fröhlich-Reiterer, Department of Paediatrics, Medical University Graz, Auenbruggerplatz 34, Graz A-8036, Austria; elke.froehlich-reiterer@ medunigraz.at}

EEF-R and JR Joint first authors and contributed equally.

Received 16 April 2013 Revised 1 April 2014 Accepted 14 April 2014 Published Online First 8 May 2014

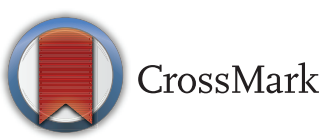

To cite: FröhlichReiterer EE, Rosenbauer J, Bechtold-Dalla Pozza S, et al. Arch Dis Child 2014;99:738-743.

\section{ABSTRACT}

Objective Increased weight gain has been reported prior to disease onset (accelerator hypothesis) and as a side effect of intensified insulin therapy in type 1 diabetes (T1D). Paediatric studies are complicated by the age-dependency and gender-dependency of BMI, and also by a trend towards obesity in the general population. The aim of this study was to evaluate factors related to the increase in $\mathrm{BMI}$ during the course of diabetes in children and adolescents with T1D in a large multicentre survey.

Design Within the DPV database (Diabetespatienten Verlaufsdokumentation) a standardised, prospective, computer-based documentation programme, data of 53108 patients with T1D, aged $<20$ years, were recorded in 248 centres. 12774 patients (53\% male, mean age $13.4 \pm 3.9$, mean diabetes duration 4.7 \pm 3.0 years and mean age at diabetes onset 8.7 \pm 4.0 years) were included in this analysis. Populationbased German reference data were used to calculate BMI-SDS and define overweight and obesity.

Results $12.5 \%$ of T1D patients were overweight and $2.8 \%$ were obese. Multiple longitudinal regression analysis revealed that female gender, low BMI at diabetes onset, intensified insulin therapy and higher insulin dose, as well as pubertal diabetes onset, long diabetes duration and onset in earlier calendar years among girls, were related to higher BMI-SDS increase during the course of diabetes ( $p<0.01$; all).

Conclusions Intensified insulin regimen is associated with weight gain during T1D treatment, in addition to demographic variables. Optimisation of diabetes management, especially in females, might limit weight gain in order to reduce overweight and obesity together with comorbidities among paediatric T1D patients.

\section{INTRODUCTION}

An increasing prevalence of obesity among youth has been demonstrated across all age groups and ethnicities. $^{1-3}$ Information about the prevalence of overweight and obesity as well as risk factors for obesity in children with type 1 diabetes (T1D) is limited and inconsistent. Furthermore, paediatric studies are complicated by the age-dependence and gender-dependence of BMI, and also by a trend towards obesity in the general population during recent years in many countries.

\section{What is already known on this topic}

The increasing prevalence of overweight and obesity and associated risk factors among youth are major global health problems. As children and adolescents with T1D are also at increased risk to develop overweight and obesity, weight gain is an important aspect in the care of children and adolescents with T1D.

\section{What this study adds}

In the present study, we identified risk factors for the increase in BMI during the course of diabetes. Female gender, low BMI at diabetes onset, intensified insulin therapy and higher insulin dose, as well as pubertal diabetes onset, long diabetes duration and onset in earlier calendar years in girls were related to higher BMI-SDS increase. Since there are some modifiable risk factors, physicians should consider these factors in their treatment regimens and educational programmes.

Weight gain has been reported to occur prior to disease onset of T1D, as the accelerator hypothesis predicts in a population with increasing weight a decreasing age at onset of diabetes, and a higher BMI-SDS in the youngest children. ${ }^{4}$ Furthermore, several studies have shown a tendency for a greater increase of weight compared with height in children with T1D as they move to puberty. ${ }^{6} 7$

Regarding body composition, studies have demonstrated a progressive increase in body fat mass, particularly in girls. ${ }^{6} 8$ The increase of weight, BMI and body fat in children and adolescents with T1D appear to be side effects of intensified insulin treatment, and seem to be related to insulin dose and number of daily injections. ${ }^{69}$

As obesity in T1D has important medical, psychological, and social implications for patients, the aim of this study was to evaluate predictors for increasing BMI in children and adolescents with T1D during the course of diabetes in a large multicentre study using data from the DPV (Diabetes Patienten Verlaufsdokumentation/multicentre 
electronic diabetes patient documentation used in diabetes centres in Austria and Germany) database.

\section{RESEARCH, DESIGN AND METHODS}

Based on the continuous diabetes data acquisition system for prospective surveillance (DPV), an observational multicentre survey was designed.

Participating centres enter data about their patients at diabetes onset or at the time-point when the patient moves to the participating centre. Follow-up data are entered into the database at each visit. Anonymous longitudinal data are transmitted for central validation and analysis twice yearly.

Inconsistent data are reported back to the centres for correction and then re-entered into the database. ${ }^{10}$

According to the guidelines of the German Diabetes Association, all centres are requested to document weight, height, blood pressure, insulin treatment (number of injections per day or pump therapy), and HbA1c levels at least once every 6 months.

From January 1995 to March 2011, data from 53108 children and adolescents with type 1 diabetes mellitus under the age of 20 years, who were treated in 248 diabetes centres from Germany $(n=235)$ and Austria $(n=13)$, were collected within the DPV initiative.

Inclusion criteria for the present analysis were: T1D for more than 6 months, documented BMI at diabetes onset (median of BMI measured during the first 3 months to correct for weight loss due to dehydration and metabolic decompensation prior to diagnosis) and documented BMI and insulin therapy during follow-up, and negative antibody screening for coeliac disease. Furthermore, patients with migration background (at least one parent born outside of Germany or Austria) were excluded, because the German/Austrian BMI reference data cannot be applied. For each patient, time-dependent data were averaged by year of diabetes duration prior to analysis; 12774 patients with a total of 54471 patient-years documented fulfilled these criteria and were included into the analysis (figure 1).

Insulin therapy was categorised as conventional therapy (CT) (one to three daily injection time-points), MDI (four or more injection time-points per day), or CSII.

Patients were classified into four groups according to age at diagnosis $(0-<5$ years, $5-<10$ years, $10-<15$ years, $15-$ 20 years) and according to diabetes duration ( $<2$ years, 2$<5$ years, $5-<10$ years, $\geq 10$ years). Because of the possible influence of the honeymoon period, we decided to define a

$n=53,108$

$n=20,468$

$\mathrm{n}=17,291$

$n=15,585$

$n=12,774$

\section{All patients}

- undocumented BMI at onset excluded

- migration background excluded

- Celiac disease excluded

-Diabetes duration $<6$ months excluded

\section{Study population}

group with 6 months to 2 years duration, a group with diabetes duration 2-5 years and, thereafter, we choose 5-year intervals.

\section{Demographic data}

BMI was derived from weight in kilograms divided by squared height in metres. Age and sex-specific BMI-reference values of 17641 children and adolescents from the population based German KIGGS study (Kinder- und Jugend-Gesundheitssurvey) were used to calculate BMI-SDS values by applying the LMS method (Box-Cox-Transformation according to Cole et al). ${ }^{11} 12$

Based on German AGA (Working group on adiposity in children and adolescents) criteria, overweight was defined as BMI between the 90th and 97th centile of the reference cohort, and obesity was defined as BMI above the 97 th centile. ${ }^{11}$ For descriptive analysis, weight change was defined as the difference between BMI-SDS at last follow-up, and baseline BMI-SDS.

\section{Glycaemic control}

HbA1c was measured as a marker of glycaemic control. To correct for different laboratory methods, HbA1c levels were mathematically standardised to the Diabetes Control and Complications Trial (DCCT) reference range of 4.05-6.05\% (21-43 mmol/mol) using the MOM (multiple of the mean) transformation. ${ }^{13}$

\section{Statistical methods}

Descriptive statistics (median, lower and upper quartile, percentage) were calculated.

To assess predictors of weight change during the course of diabetes, we performed longitudinal regression analysis with BMI-SDS as dependent variable adjusting for baseline BMI-SDS. In detail, linear mixed longitudinal regression models were applied using a repeated measurement design with a compoundsymmetry covariance structure for BMI-SDS in order to account for within-patient dependency of the outcome. First, we performed 'univariable' longitudinal regression for each potential influencing factor adjusting for onset-BMI-SDS. Then we build a first multivariable longitudinal model including all effects and interaction terms of these with sex. Subsequently, all effects significant in this multivariable model were included in a final model to estimate effects sizes on BMI-SDS. Age at onset, diabetes duration, sex, therapy regimen $(1-2,3,4$ or more daily injections, continuous subcutaneous insulin infusion (CSII)), daily insulin dose (IU per kg bodyweight), use of short-acting or long-acting analogues, baseline BMI-SDS and year of manifestation were included as fixed effects. In all models, treatment centre was included as a random effect in order to adjust for between-centre variation. Estimates of effect sizes (regression coefficients) with 95\% CIs and respective Wald and F-tests were used to assess the importance of predictors (table 2). Further, results are presented as adjusted means (least squares means) ( $p$ values) (figure 2). Multivariable adjusted means for fixed effects were estimated using observed marginal frequencies for categorical variables (age at onset, diabetes duration, sex and insulin regimen, use of regular insulin), a daily insulin dose of $0.8 \mathrm{IU} / \mathrm{kg}$, BMI-SDS at onset of 0.2 , and diabetes diagnosis in the year 2000). CIs of adjusted means and comparisons between categories for fixed effects ( $p$ values and CIs) were adjusted for multiple comparisons according to Bonferroni or Tukey, respectively, when three or more categories were present. A two-sided $\mathrm{p}$ value less than 0.05 was considered statistically significant.

Data were analysed using the SAS software (V.9.4; SAS Institute, Cary, North Carolina, USA).

Figure 1 Patient selection from the database. 

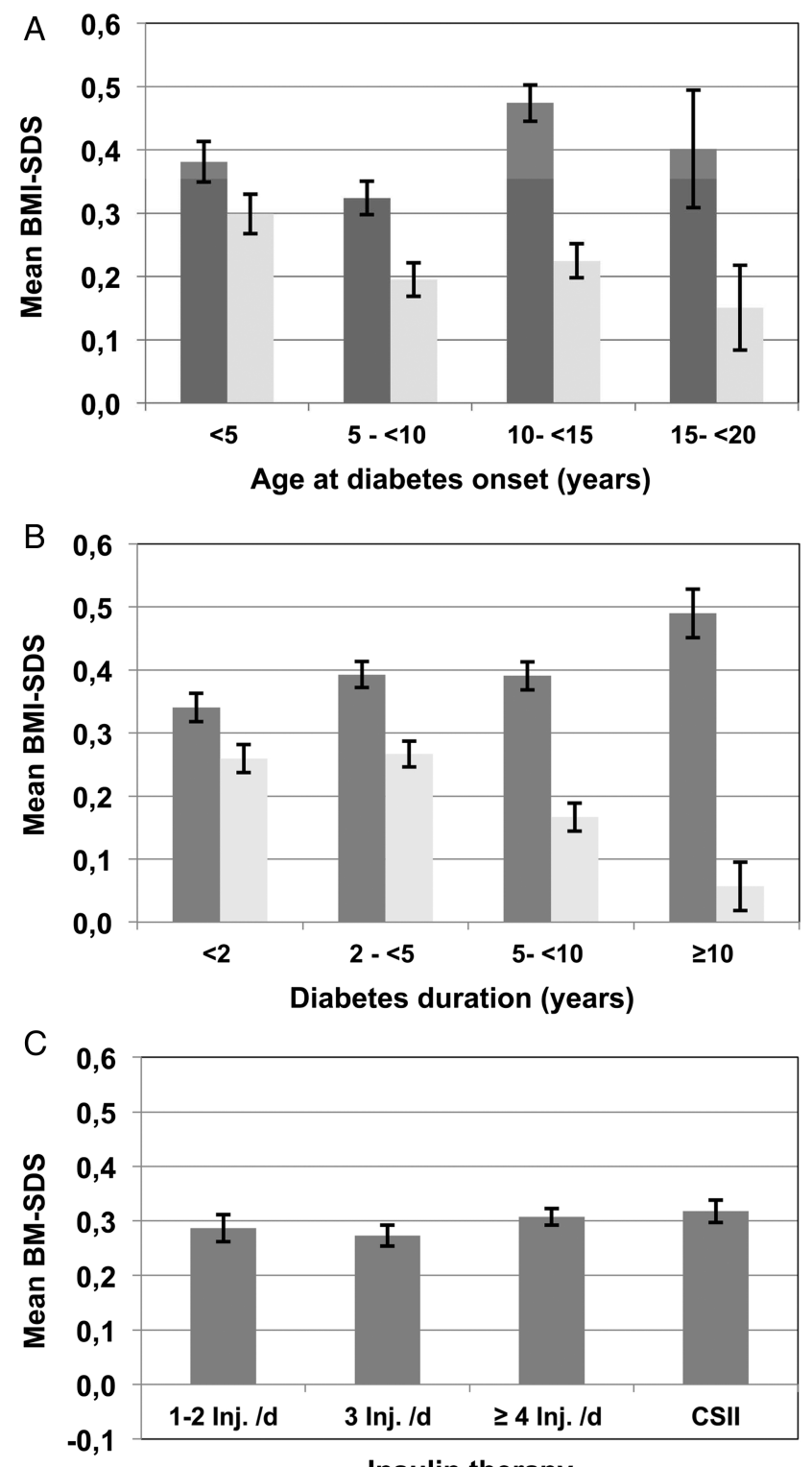

Insulin therapy

Figure 2 Adjusted mean BMI-SDS (and simultaneous 95\% Cls, adjusted according to Bonferroni) according to age at diabetes onset, diabetes duration (males: light grey bars, and females: dark grey bars) and insulin therapy estimated from multivariable longitudinal regression model in table 2.

\section{RESULTS}

Baseline characteristics

Data from 54471 documented patient-years of 12774 patients (53\% male) who fulfilled the inclusion criteria were analysed. Mean duration of follow-up (corresponding to current diabetes duration) was $4.7 \pm 3.0$ years. At the end of follow-up, $12.5 \%$ were overweight and $2.8 \%$ were classified as obese. Demographic data for the study group and each sex are summarised in table 1 .

\section{Predictors of BMI change during the course of diabetes}

Results of the univariable longitudinal regression model demonstrate, that BMI-SDS increase was significantly related to female gender, age at diabetes onset, diabetes duration, intensified insulin therapy ( $\geq 4$ injection time-points or CSII), higher insulin dose, low BMI-SDS at diabetes onset, the use of short-acting and long-acting (inverse association) insulin analogues $(\mathrm{p}<0.001$, all except long-acting insulin, $\mathrm{p}=0.004)$ (table 2).

After simultaneously including all effects and interaction terms of these with sex in a first multivariable longitudinal regression model, the main effects sex, age at onset, diabetes duration, insulin regimen, insulin dose, BMI-SDS at onset, use of shortacting analogues $(<0.001$, all), use of long-acting analogues $(p=0.001)$ and calendar year at diabetes onset $(p=0.004)$, and the interactions of sex with age at onset, diabetes duration, calendar year, and use of insulin analogues $(<0.001$, all $)$ remained significant, as well as the interaction term of insulin dose and sex $(p=0.035))$, and were included in the final multivariable model to estimate adjusted effect sizes (table 2).

Results of the final model confirmed that sex, age at onset, diabetes duration, insulin regimen, BMI-SDS at onset, insulin dose, calendar year (only in females) as well as use of shortacting (only in females) and long-acting analogues (only in males) were significant predictors of weight gain.

BMI-SDS was significantly higher in females (table 2).

Comparing the four age groups at diabetes onset, we found that the increase in BMI-SDS was highest in females in the age group $10-<15$ years compared to the age groups $15-20$ years, $5-<10$ years and $<5$ years $(p=0.232,<0.001,<0.001)$. Further, weight gain among females in the group $5-<10$ years was significantly lower compared to the groups $<5$ years $(\mathrm{p}<0.001)$ and $10-<15(\mathrm{p}<0.001)$ (table 2, figure 2A). Among males, weight gain was significantly higher in the group with diabetes onset $<5$ years compared with all other groups $(\mathrm{p}<0.001$, all $)$. Further, weight gain in the onset group 1520 years was significantly lower than in males with onset in the ages $10-<15$ years $(\mathrm{p}=0.039)$.

Comparing the four groups of diabetes duration, results showed that weight gain in males was significantly higher in the groups $<2$ and $2-<5$ years compared to the other duration groups $(\mathrm{p}<0.001$, all). There was no difference between $<2$ and $2-<5$ years diabetes duration $(\mathrm{p}=0.572)$, weight gain in the group with $10-<15$ years duration was significantly higher than in the group $\geq 10$ years $(p<0.001)$ (table 2 , figure $2 B$ ).

In females, weight gain was highest in the group $\geq 10$ years diabetes duration, and the differences were significant between all groups $(<0.001$, all $)$, with the exception that we found no difference between the group $2-<5$ and $5-<10$ years $(p=0.988)$.

Weight gain was highest in patients with pump therapy, independently of sex (table 2, figure 2C). BMI-SDS increase in this group was significantly higher than in patients with three injections $(p<0.001)$ or two injections $(p=0.029)$, but there was no difference between pump therapy and $\geq 4$ injections $(p=0.463)$. Furthermore, patients with intensified injection therapy $(\geq 4$ injections) showed a significant higher weight gain compared to patients with three injections $(p<0.001)$. Increase in BMI-SDS did not significantly differ between the group with 1-2 injections daily and the group with three daily injections) $(\mathrm{p}=0.367)$.

Low BMI-SDS at baseline and higher insulin dose was associated with a significantly higher weight gain independent of sex. Diabetes onset in earlier calendar years was associated with a higher weight gain in girls (table 2).

\section{CONCLUSIONS}

The increasing prevalence of overweight and obesity and associated risk factors among youth are major global health problems. As children and adolescents with T1D are also at increased risk to develop overweight and obesity, weight gain is 
Table 1 Demographic data of the study group $(n=12774)$

\begin{tabular}{|c|c|c|c|}
\hline & Total & Male & Female \\
\hline $\mathrm{N}(\%)$ & 12774 & $6816(53.4)$ & $5958(46.6)$ \\
\hline Current age (years) & 14.3; (10.8; 16.9) & 14.6; $(10.8 ; 17.0)$ & 14.0; (10.7; 16.6) \\
\hline Age at diabetes onset (years) & $9.0 ;(5.5 ; 11.9)$ & $9.2 ;(5.6 ; 12.4)$ & $8.7 ;(5.4 ; 11.4)$ \\
\hline Current diabetes duration (years) & $4.0 ;(2.3 ; 6.5)$ & $3.9 ;(2.2 ; 6.3)$ & $4.2 ;(2.3 ; 6.7)$ \\
\hline \multicolumn{4}{|l|}{ Current therapy (\%) } \\
\hline$\leq 2$ Injections/d & 2.5 & 2.6 & 2.4 \\
\hline 3 Injections/d & 7.0 & 7.2 & 6.8 \\
\hline$\geq 4$ Injections/d & 60.8 & 63.6 & 57.6 \\
\hline CSII & 28.6 & 25.5 & 32.2 \\
\hline BMI-SDS baseline & $0.19 ;(-0.42 ; 0.80)$ & $0.23 ;(-0.39 ; 0.84)$ & $0.15 ;(-0.45 ; 0.75)$ \\
\hline Current BMI-SDS & $0.32 ;(-0.24 ; 0.88)$ & $0.26 ;(-0.30 ; 0.80)$ & $0.42 ;(-0.16 ; 0.95)$ \\
\hline Current percentage overweight (\%) & 12.5 & 11.3 & 13.8 \\
\hline Current percentage obesity (\%) & 2.8 & 2.6 & 3.2 \\
\hline BMI SDS increase & $0.11 ;(-0.28 ; 0.53)$ & $0.03 ;(-0.37 ; 0.41)$ & $0.22 ;(-0.17 ; 0.67)$ \\
\hline BMI SDS increase $>0.5(\%)$ & 26.4 & 20.2 & 33.5 \\
\hline Current $\mathrm{HbA} 1 \mathrm{c}(\%)(\mathrm{n}=12720)(\mathrm{mmol} / \mathrm{mol})$ & $\begin{array}{l}7.7 ;(7.0 ; 8.7) \\
61 ;(53 ; 72)\end{array}$ & $\begin{array}{l}7.7 ;(7.0 ; 8.6) \\
61 ;(53 ; 70)\end{array}$ & $\begin{array}{l}7.7 ;(7.0 ; 8.7) \\
61 ;(53 ; 72)\end{array}$ \\
\hline
\end{tabular}

Data are median and lower and upper quartile or percentage. Current data refer to the last follow-up of each patient.

an important aspect in the care of children and adolescents with T1D. In the present study, we identified risk factors for the increase in BMI during the course of diabetes using the DPV database.
Especially girls, diagnosed with T1D at an age between 1015 years (around puberty), with CSII, and lower BMI at diabetes onset and earlier years of manifestation increased their BMI during the course of diabetes.

Table 2 Predictors of BMI-SDS

\begin{tabular}{|c|c|c|c|c|c|c|}
\hline \multirow[b]{3}{*}{ Effect } & \multicolumn{2}{|l|}{ Univariable regression* } & \multicolumn{4}{|l|}{ Multivariable regressiont } \\
\hline & \multirow[b]{2}{*}{ Effect size estimate $(95 \% \mathrm{Cl})$} & \multirow[b]{2}{*}{$p$ Value } & \multicolumn{2}{|l|}{ Male } & \multicolumn{2}{|l|}{ Female } \\
\hline & & & $\begin{array}{l}\text { Effect size estimate } \\
(95 \% \mathrm{Cl})\end{array}$ & $\mathrm{p}$ Value & Effect size estimate $(95 \% \mathrm{Cl})$ & p Value \\
\hline Age at onset & & $<0.001$ & & $<0.001$ & & $<0.001$ \\
\hline $15-\leq 20$ & $-0.106(-0.151$ to -0.061$)$ & $<0.001$ & $-0.148(-0.222$ to -0.074$)$ & $<0.001$ & $0.021(-0.079$ to 0.121$)$ & 0.951 \\
\hline $10-<15$ & $0.004(-0.018$ to 0.026$)$ & $<0.708$ & $-0.074(-0.113$ to -0.036$)$ & $<0.001$ & $0.093(0.051$ to 0.135$)$ & $<0.001$ \\
\hline $5-<10$ & $-0.082(-0.103$ to -0.060$)$ & $<0.001$ & $-0.104(-0.142$ to -0.066$)$ & $<0.001$ & $-0.057(-0.096$ to -0.018$)$ & 0.001 \\
\hline$<5$ (ref.) & 0 & - & 0 & - & 0 & - \\
\hline Diabetes duration & & $<0.001$ & & $<0.001$ & & $<0.001$ \\
\hline$\geq 10$ years (ref.) & 0 & - & 0 & - & 0 & - \\
\hline $5-<10$ years & $-0.008(-0.027$ to 0.010$)$ & 0.365 & 0.110 (0.076 to 0.144$)$ & $<0.001$ & $-0.099(-0.134$ to -0.064$)$ & $<0.001$ \\
\hline $2-<5$ years & 0.031 (0.012 to 0.049$)$ & 0.001 & $0.210(0.174$ to 0.246$)$ & $<0.001$ & $-0.097(-0.134$ to -0.060$)$ & $<0.001$ \\
\hline$<2$ years & $-0.016(-0.035$ to 0.003$)$ & 0.109 & 0.203 (0.163 to 0.242 ) & $<0.001$ & $-0.149(-0.190-0.109)$ & $<0.001$ \\
\hline \multicolumn{7}{|l|}{ Sex } \\
\hline Male (ref.) & 0 & - & \multicolumn{4}{|c|}{0} \\
\hline Female & 0.123 (0.107 to 0.139$)$ & $<0.001$ & \multicolumn{4}{|c|}{$0.149(0.130$ to 0.168$) \ddagger \quad<0.001$} \\
\hline Insulin regimen & & $<0.001$ & \multicolumn{4}{|c|}{$<0.001$} \\
\hline CSII (ref) & 0 & - & \multicolumn{4}{|c|}{0} \\
\hline 4+injections & $-0.028(-0.040$ to -0.017$)$ & $<0.001$ & $-0.010(-0.029$ to 0.008$) \S$ & 0.463 & & \\
\hline 3 injections & $-0.062(-0.077$ to -0.048$)$ & $<0.001$ & $-0.045(-0.068$ to -0.022$)$ & $<0.001$ & & \\
\hline$\leq 2$ injections & $-0.050(-0.068$ to -0.031 & $<0.001$ & $-0.031(-0.060$ to -0.002$)$ & 0.029 & & \\
\hline Insulin dose (IU/kg) & $0.059(0.042$ to 0.077$)$ & $<0.001$ & $0.086(0.059$ to 0.113$)$ & $<0.001$ & $0.042(0.014$ to 0.070$)$ & 0.003 \\
\hline BMI-SDS at baseline & $0.676(0.667$ to 0.684$)$ & $<0.001$ & \multicolumn{4}{|c|}{$-0.318(-0.327$ to -0.309$) \S \quad<0.001$} \\
\hline Calendar year at onset & $-0.002(-0.004$ to 0.0001$)$ & 0.068 & $0.0009(-0.002$ to 0.003$)$ & 0.516 & $-0.007(-0.009$ to -0.004$)$ & $<0.001$ \\
\hline Use of short-acting analogue & $0.030(0.021$ to 0.039$)$ & $<0.001$ & $0.007(-0.007$ to 0.021$)$ & 0.358 & $0.047(0.032$ to 0.061$)$ & $<0.001$ \\
\hline Use of long-acting analogue & $-0.014(-0.024$ to -0.005$)$ & 0.004 & $-0.037(-0.051$ to -0.022$)$ & $<0.001$ & $0.001(-0.014$ to 0.017$)$ & 0.883 \\
\hline
\end{tabular}

${ }^{*}$ Separate model for each effect adjusting for BMI-SDS at baseline, per unit increase for continuous variables.

tIndependent effects in the model: age at onset, diabetes duration, sex, insulin regimen, insulin dose, BMI-SDS at baseline, calendar years at onset, use of short-acting analogue, use of long-acting analogue and interaction terms between sex and age at onset, diabetes duration, insulin dose, calendar year at onset and use of short-acting and long-acting analogue. ‡Estimated from least squares means.

$\S$ No significant difference between sexes. 
The 18 years follow-up of the Pittsburgh Epidemiology of Diabetes Complications Study (EDC) performed in adults did not find any gender differences in overweight or weight gain. ${ }^{14}$ This finding is in contrast with our study, as we found a significantly higher increase in BMI-SDS in girls compared to boys. The gender difference in BMI might be the result of a greater weight gain during puberty in girls compared to boys due to alterations in GH/IGF-1 axis in patients with T1D, the influence of sex steroids, ${ }^{6}$ and the higher insulin resistance in girls compared to boys. ${ }^{15}$

Puberty is, therefore, a risk factor for the increase in BMI, as our data show that diabetes onset at the age between 10 and 15 years (around puberty) was significantly associated with an increase in BMI in females.

Puberty is characterised by physiological insulin resistance, and adolescents with T1D are even more insulin resistant than healthy controls. $^{16}$ Girls accumulate more fat mass during puberty, whereas boys gain more lean body mass. ${ }^{6}$ Girls with T1D followed longitudinally during puberty display a high BMI at Tanner stage 5 , which is associated with a higher percentage of fat mass. ${ }^{17}$ Furthermore, Codner $e$ al $^{18}$ reported that BMI-SDS was lower for Tanner stages 1 and 3 in girls with T1D compared to healthy controls, which is in line with recently published data, ${ }^{15}$ but throughout puberty, girls with T1D had a greater increase in BMI-SDS compared to healthy controls. ${ }^{18}$ Thus, puberty and insulin resistance seem to be critical factors for weight gain in girls.

Intensive insulin therapy was identified as another important risk factor for the increase in BMI in our study. Although intensive insulin therapy has been shown to lower the development of microvascular and ultimately macrovascular complications in patients with T1D, ${ }^{19}{ }^{20}$ its beneficial effects may be partially offset by its weight gain-promoting properties, which are paralleled by the occurrence of obesity-associated cardio-metabolic risk factors. ${ }^{21} 22$ There are several studies in the literature showing that a more intensive therapy (multiple daily injection therapy or CSII) leads to an increase in weight and BMI. ${ }^{9} 162324$ In our study, we also found that the intensity of insulin therapy, especially CSII, was associated with an increase in BMI-SDS, and weight gain was highest in patients with pump therapy, independent of sex.

Higher insulin dose was another risk factor for the increase in BMI, particularly in males. In our study, we found that the use of short-acting insulin analogues was associated with a higher increase in BMI in girls, but not in boys. By contrast, the use of long-acting insulin analogues was associated with a reduced gain in BMI-SDS in boys, but not in girls. Therefore, our data suggest that the number of injections, the amount of insulin and type of insulin, are driving factors for BMI increase. The reasons for this are probably the anabolic effect of insulin, as insulin itself promotes weight gain by stimulating lipogenesis, inhibiting protein catabolism and slowing basal metabolism ${ }^{25}$ on the one hand, and on the other hand, weight gain is influenced by caloric intake.

Children with intensive insulin therapy are likely to have a more flexible lifestyle and they are experienced in adjusting insulin dose to carbohydrate consumption. Therefore, they are supposed to face less food restrictions and might take more meals with higher energy content compared to patients on CT, who are advised to eat fixed amount of carbohydrates at fixed times of the day. One would assume that the use of short-acting insulin analogues might be a risk factor for the increase in BMI, as patients are allowed to inject short-acting insulin analogues more often. However, our data confirm this assumption only in girls. The opposite might also be true: data on nutrition in T1DM are scanty, but there are studies showing that weight gain might also be influenced by high-fat diets, as youth with diabetes consumed fewer carbohydrates than matched controls to avoid injections. ${ }^{26} 27$

The strength of our study is the multicentre approach, the long-term, standardised follow-up of a large number of children and adolescents with T1D and the use of longitudinal regression models. Furthermore, height and weight are well-standardised parameters in the DPV database, as well as insulin regimen.

Limitations of our study are that insulin doses were selfreported from patients/parents, and that we do not have data on BMI of the family members, or on daily caloric intake of the patients. Furthermore, height and weight for the reference dataset were collected within the KIGGS study during 20032006. As data from the DPV system cover the time period from 1995 to 2013, the KIGGS data cover the middle of the study period. Some overestimation or underestimation in BMI-SDS due to secular trends, however, cannot be excluded for patient data beyond 2006 or prior to 2003.

In conclusion, female gender, diabetes manifestation during puberty in girls, and intensified insulin therapy and insulin dose significantly influence the increase in BMI during the course of diabetes. Therefore, one of the aims in the care of children and adolescents with T1D is to explain these risk factors to patients and parents to avoid excessive increase in BMI, especially during puberty. Intervention programmes (eg, sport programmes) and dietary education should start early.

Acknowledgements For a full list of the centres participating in the DPV initiative and contributing their data to this study please see the online supplementary file on our website.

Contributors EEF-R researched data, wrote the manuscript. SB-DP researched data reviewed/edited the manuscript and contributed to the discussion. SEH researched data, reviewed/edited the manuscript and contributed to the discussion. JR researched data, reviewed/edited the manuscript, contributed to the discussion and analysed the data. ES reviewed/edited the manuscript and contributed to the discussion. RWH researched data, reviewed/edited the manuscript and contributed to the discussion.

Funding The DPV initiative is financially supported by the German Ministry of Health, the German Diabetes Foundation, the German Diabetes Association, the BMBF competence network for diabetes mellitus (FKZ: 01GI1106) and competence net obesity (FKZ: 01GI1130), excellence centre 'Metabolism' of the state of Baden Württemberg, the Dr Bürger-Büsing Foundation and the European Foundation for the study of diabetes (EFSD).

\section{Competing interests None.}

Provenance and peer review Not commissioned; externally peer reviewed.

\section{REFERENCES}

1 Freedmann DS, Khan LK, Serdula MK, et al. Racial and ethnic differences in secular trends for childhood BMI, weight and height. Obesity 2006;14:301-8.

2 Ogden CL, Carroll MD, Kit BK, et al. Prevalence of obesity and trends in body mass index among US children and adolescents, 1999-2010. JAMA 2012;307:483-90.

3 Livingstone B. Epidemiology of childhood obesity in Europe. Eur J Pediatr 2000;159 (Suppl 1):14-34.

4 Wilkin TJ. The accelerator hypothesis: weight gain as the missing link between Type I and Type || diabetes. Diabetologia 2001;44:914-22.

5 Knerr I, Wolf J, Reinehr T, et al. The 'accelerator hypothesis': relationship between weight, height, body mass index and age at diagnosis in a large cohort of 9,248 German and Austrian children with type 1 diabetes mellitus. Diabetologia 2005;48:2501-4.

6 Dunger D, Ahmed L, Ong K. Growth and body composition in type 1 diabetes mellitus. Horm Res 2002;58(Suppl 1):66-71.

7 Holl RW, Grabert M, Sorgo W, et al. Contributions of age, gender and insulin administration to weight gain in subjects with IDDM. Diabetologia 1998;41:542-7.

8 Ahmed ML, Ong K, Watts AP, et al. Elevated leptin levels are associated with excess gains in fat mass in girls, but not boys, with type 1 diabetes: Longitudinal study during adolescents. J Clin Endocrinol Metab 2001;86:1188-93.

9 Holl RW, Swift PGF, Mortensen HB, et al. Insulin injection regimens and metabolic control in an international survey of adolescents with type 1 diabetes over 3 years: result from the Hvidoere study group. Eur J Pediatr 2003;162:22-9. 
10 Grabert M, Schweiggert F, Holl RW. A framework for diabetes documentation and quality management in Germany: 10 years of experience with DPV. Comput Methods Programs Biomed 2002;69:115-21.

11 Kurth BM, Schaffrath Rosario A. [The prevalence of overweight and obese children and adolescents living in Germany. Results of the German Health Interview and Examination Survey for Children and Adolescents (KiGGS)]. Bundesgesundheitsblatt Gesundheitsforschung Gesundheitsschutz 2007:50:736-43.

12 Cole TJ, Green PJ. Smoothing reference centile curves: the LMS method and penalized likelihood. Stat Med 1992;11:1305-19.

13 Schütt M, Kern W, Krause U, et al. Is the frequency of self blood glucose measurements related to longterm metabolic control? Multicenter analysis including 24,500 patients from 191 centers in Germany and Austria. Exp Clin Endocrinol Diabetes 2006;114:384-8.

14 Conway B, Miller RG, Costacou T, et al. Temporal patterns in overweight and obesity in type 1 diabetes. Diabet Med 2010;27:398-404.

15 Murphy MJ, Metcalf BS, Voss LD, et al. Early Bird Study (Early Bird 6). Girls at five are intrinsically more insulin resistant than boys: the programming hypotheses revisited-The Early Bird Study (EarlyBird 6). Pediatrics 2004;113:82-6.

16 Dunger DB. Diabetes in puberty. Arch Dis Child 1992;75:569-70.

17 Ahmed ML, Connors MH, Drayer NM, et al. Pubertal growth in IDDM is determined by HbA1c levels, sex and bone age. Diabetes Care 1998; 21:831-5

18 Codner E, Barrera A, Mook-Kanamori D, et al. Ponderal gain, waist-to-hip ratio, and pubertal development in girls with type-1 diabetes mellitus. Pediatr Diabetes 2004:5:182-9.

19 The Diabetes Control and Complication Trial Research Group. The effect of intensive treatment of diabetes on the development and progression of long-term complications in insulin-dependent diabetes mellitus: the Diabetes Control and Complication Trial Research Group. N Engl J Med 1993;329:977-86.

20 Nathan DM, Cleary PA, Backlund JY, et al. Diabetes Control and Complications Trial/Epidemiology of Diabetes Interventions and Complications (DCCT/EDIC) Study Research Group. Intensive diabetes treatment and cardiovascular disease in patients with type 1 diabetes. N Engl J Med 2005;353:2643-53.

21 Van Vliet M, Van der Heyden JC, Diamant M, et al. Overweight is highly prevalent in children with type 1 diabetes and associates with cardiometabolic risk. J Pediatr 2010;156:923-9.

22 Schwab KO, Doerfer J, Marg W, et al. DPV Science Initiative and the Competence Network Diabetes mellitus. Characterization of 33488 children and adolescents with type 1 diabetes based on the gender-specific increase of cardiovascular risk factors. Pediatr Diabetes 2010;11:357-63.

23 Ludvigsson J, Samuelsson U. Continuous insulin infusion (CSII) or modern type of multiple daily injections (MDI) in diabetic children and adolescents: a critical review on a controversial issue. Pediatr Endocrinol Rev 2007:5:666-78.

24 Jakisch BI, Wagner VM, Heidtmann B, et al. German/Austrian DPV Initiative and Working Group for Paediatric Pump Therapy. Comparison of continuous subcutaneous insulin infusion (CSII) and multiple daily injections (MDI) in paediatric Type 1 diabetes: a multicentre matched-pair cohort analysis over 3 years. Diabet Medicine 2008:25:80-5.

25 Nair K, Hallisay D, Garrow J. Increased energy expenditure in poorly controlled type 1 (insulin-dependent) diabetic patients. Diabetologia 1984;27:13-16.

26 Patton SR. Adherence to diet in youth with type 1 diabetes. J Am Diet Assoc 2011:111:550-5

27 Meissner T, Wolf J, Kersting M, et al. Carbohydrate intake in relation to BMI, HbA1c and lipid profile in children and adolescents with type 1 diabetes. Clin Nutr 2014;33:75-8. 


\section{ADC Predictors of increasing BMI during the course of diabetes in children and adolescents with type 1 diabetes: data from the German/Austrian DPV multicentre survey}

Elke E Fröhlich-Reiterer, Joachim Rosenbauer, Susanne Bechtold-Dalla Pozza, Sabine E Hofer, Edith Schober, Reinhard W Holl and on behalf of the DPV-Wiss Study Group and the German BMBF Competence Networks Diabetes mellitus and Obesity

Arch Dis Child 2014 99: 738-743 originally published online May 8, 2014 doi: 10.1136/archdischild-2013-304237

Updated information and services can be found at:

http://adc.bmj.com/content/99/8/738

These include:

Supplementary Supplementary material can be found at:

Material http://adc.bmj.com/content/suppl/2014/05/09/archdischild-2013-3042 37.DC1

References This article cites 27 articles, 2 of which you can access for free at: http://adc.bmj.com/content/99/8/738\#BIBL

Email alerting Receive free email alerts when new articles cite this article. Sign up in the service box at the top right corner of the online article.

Topic
Collections

Articles on similar topics can be found in the following collections

Child health (3922)

Adolescent health (329)

Health education (555)

Health promotion (611)

Obesity (nutrition) (325)

Obesity (public health) (325)

Diabetes (339)

Immunology (including allergy) (2018)

Metabolic disorders (761)

\section{Notes}

To request permissions go to:

http://group.bmj.com/group/rights-licensing/permissions

To order reprints go to:

http://journals.bmj.com/cgi/reprintform

To subscribe to BMJ go to:

http://group.bmj.com/subscribe/ 